\title{
ABC Classification of Risk Factors in Production Supply Chains with Uncertain Data
}

\author{
Aleksandar Vujović, ${ }^{1}$ Aleksandar Đorđević, ${ }^{2}$ Ranka Gojković, ${ }^{3}$ and Milan Borota ${ }^{4}$ \\ ${ }^{1}$ Faculty of Mechanical Engineering, University of Montenegro, Podgorica, Montenegro \\ ${ }^{2}$ Higher Technical School of Professional Studies Zvečan, Zvečan, Serbia \\ ${ }^{3}$ Faculty of Mechanical Engineering, University of East Sarajevo, East Sarajevo, Bosnia and Herzegovina \\ ${ }^{4}$ Merkur, Belgrade, Serbia \\ Correspondence should be addressed to Aleksandar Đorđević; adjordjevic@kg.ac.rs
}

Received 6 July 2017; Accepted 24 July 2017; Published 28 August 2017

Academic Editor: Anna M. Gil-Lafuente

Copyright (C) 2017 Aleksandar Vujović et al. This is an open access article distributed under the Creative Commons Attribution License, which permits unrestricted use, distribution, and reproduction in any medium, provided the original work is properly cited.

\begin{abstract}
The problem of classification of risk factors in an uncertain environment is part of the risk management problem, which has a critical effect on the competitive advantage of production supply chain. The severities of consequences, their relative importance, and the frequency of occurrence of risk factors are defined by risk management team, depending on their experience and the results of good practice. Fuzzy rating of the severities of consequences and the frequency of occurrence of risk factors are described by linguistic expressions, which are modeled by triangular fuzzy numbers. The risk values, obtained by the materialization of the identified risk factors, are given precisely with the usage of fuzzy algebra rules. The classification criterion is defined as the distance between current risk value and extreme risk values. The proposed model enables determination of the priorities of risk factors. It is illustrated by an example with real-life data from a production supply chain in auto industry.
\end{abstract}

\section{Introduction}

Due to dynamic changes in modern business environment, changes in social, economic, and demographic fields, as well as in workforce diversity, the number of possible uncertainties is constantly increasing and affecting the definition of business process strategies. Uncertainties which have the greatest influence on business process strategy are denoted as risks. In the literature, numerous definitions of risk may be found $[1,2]$. The existence of the differences in the definitions of risk is based on the existing diversity of research domains. In the past few years, theory and management practice focused on the concept of risk, which has to be managed in order to decrease risk influence on business strategy [3], especially in conditions with limited resources [4]. The process of defining risk management strategies may be based on the priorities of risk factors (RFs), which are determined according to the strength the influence of RFs on business strategies.
Risk management is a complex task, especially in production supply chains (PSCs), and it includes the identification of RFs, the analysis and the assessment of risk, and the determination of activities which should lead to risk value reduction [5-7]. The determination of appropriate management initiatives for risk value reduction has been considered from various aspects, which are briefly described in the following text. Risk analysis for each identified RF has been considered in the literature $[8,9]$. Authors have suggested different management initiatives for reduction of each calculated risk value. Some authors suggest that materialization of a number of different RFs affects value of total risk $[10,11]$. Based on these results, the value of total risk can be reduced by the application of appropriate management initiatives. In practice, risk management is usually based on certain levels of risk. Hence, in the literature, there are a large number of papers which present various methods for the assessment of risk level $[12,13]$. Management initiatives for decreasing risk level may be defined based on the obtained results. 
In the literature, there are papers in which the problem of RFs rank is presented as a multicriteria decision-making problem $[14,15]$. By applying different multicriteria optimization methods, the rank of RFs is determined. The priority of the management initiatives is based upon the obtained rank. In such cases, the focus of the management team is only at the RF which is ranked first. However, according to the best practice results, the total risk in complex PSCs depends on more than one RF. Because of that, priority of management initiatives cannot be adequately determined by applying multicriteria decision-making method. The authors think that risk management in considered PSC system should be based on the results of the classification of RFs. Torabi et al. [16] have developed the best-worst method for the classification of RFs.

According to Basu [17], if there is no proper RFs classification, then a risk of little importance may get unnecessary attention, while the vital risk may be overlooked. The lack of methods for the RFs classification under uncertainties is noticeable. Motivation for this research arises from the abovementioned facts.

The main goal of the paper is to identify and classify RFs under PSCs. According to the relevant literature data $[4,18]$, it can be said that 20 RFs are significant for a sustainable PSC management. Priority of management initiatives for risk reduction is based on the obtained classification results. This way, management time consumption and its overall costs are remarkably reduced and the sustainability of PDS is increased over a long period of time.

The uncertainty in severity of the consequences and the frequency of occurrence of RFs are described by predefined linguistic expressions, which are modeled by triangular fuzzy numbers (TFNs) [19]. The fuzzy set theory allows that all uncertainties and imprecisions of any kind be sufficiently well described quantitatively.

The paper is organized in the following manner. Section 2 presents research papers from relevant literature. The identification of RFs set in PSC, the determination of all possible consequences which may occur due to the influence of each identified RF and their relative importance, the handling of the uncertainties in severity of consequences, and the frequency of RFs occurring, as well as the proposed algorithm, are presented in Section 3. In Section 4, the proposed model is illustrated with real-life data, originating from a PSC in automotive industry from central Serbia. Conclusions are presented in Section 5.

\section{A Literature Review}

Increased uncertainty on the global market and growing use of information technology have led to the growing vulnerability of PSCs and governments and their exposure to high level of risks.

Risk management is receiving attention due to dynamic changes in business environment. Stulz [21] defined risk management as the process of planning, organizing, leading, and controlling the activities of the organization in order to reduce the effects of risk on the values of performance by which the effectiveness of an enterprise is measured. This process is implemented through a number of predefined steps $[22,23]$.

A growing number of authors and managers are interested in identifying critical RFs, especially when the resources are limited [4], as decision-making should be risk informed, not risk based. In order to accomplish a balanced and effective risk reduction strategy it is necessary to comprehend diversity and coherence of PSC risks. Literature review shows that different approaches for RFs identification in PSC are introduced, as briefly described in the following text.

The simplest procedure for identifying RFs in a PSC is the checklist. It is one of the quickest and least expensive procedures, very frequently in use. The effectiveness of this procedure depends on the knowledge and the experience of decision makers, which presents its main drawback. Many authors tried to define the lists of RFs for certain groups of enterprises. According to Chopra and Sodhi [24] all RFs in a PSC can be classified into nine groups: (1) interruptions of working processes, (2) delays of raw materials, (3) malfunctioning of information infrastructure, (4) incorrect forecast, (5) vertical integration of the PSC, (6) uncertain supply, (7) uncertain demand, (8) stocks of goods, and (9) flexibility and costs of capacity. Four different groups of RFs in a PSC may be distinguished: (1) increase of prices, (2) disrespect of the procedures for environmental protection, (3) loss of intellectual property rights, and (4) interrupts in the working process. Some authors [4] have identified and classified many RFs in PSCs: operational RFs [24], economic RFs [25], environmental RFs, and social RFs [26]. In this paper, the process of identifying RFs is literature source-based [4] and is described in detail.

Risk analysis is the procedure used to handle all the identified RFs. Risk value can be calculated as the product of the severity of the consequences that arise from the materialization of the RFs and the probability of the occurrence of RFs. In the literature, a few procedures have been proposed for the analysis of the potential consequences, such as fault trees and Petri Nets [27]. The severities of consequences assessment, for the situations where the consequences do not result in fatal injuries, can be performed in different ways. Consequences can be usually considered in terms of the overall costs, and thus their severities can be expressed in monetary units. Woodruff [28] introduces the preposition that the equation of monetary value represents the cost of one life loss. With this assumption in mind, the severities of the consequences are determined from this benchmark. Hess [29] described the severities of the consequences by a stochastic variable with Poisson distribution. Severities of consequences have been evaluated by applying statistical methods and described by crisp values [10]. Mapping the severities of consequences into monetary or some other measurement units is substantially biased with subjective assessment of decision maker. Hence, the description of severities of consequences by crisp values can be considered an insufficiently correct approach as well. Modeling the severities of consequences by a probability theory requests a sufficient number of accurate evidence data. For PSCs in uncertain and volatile market environments it is difficult to meet this requirement. Many authors think that the severities of consequences can be adequately described by 
using linguistic expressions modeling on the base of fuzzy sets theory [12, 13]. Eckert and Gatzert [10] have supposed that each RF can produce more consequences, which have equivalent relative importance. The aggregation of the severities of consequences is performed by the developed model. According to the results in practice, in this paper, it is assumed that the consequences which appear due to the materialization of each RF have different values of relative importance. The overall severity of all consequences for each RF is calculated by using Fuzzy Order Weighted Operator (FOWA) [30]. The frequency of the materialization of identified RFs is based on the evidence data. Hess [29] described the values of frequencies by a stochastic variable with Poisson distribution. In other research papers considered, the values of frequencies are described by precise numbers. With respect to the fact that PSC operates in a rapidly changing environment, the authors of this paper think that values of frequencies can be described sufficiently well by using linguistic variables, modeled by fuzzy sets.

According to Basu [17], sound RFs classification is an essential part of RFs assessment and management. Unfortunately, there are only a few papers which consider the problem of RFs classification in the literature. Classifications of other items with respect to many fuzzy criteria are considered in the literature $[31,32]$. The classification criterion is defined as the product of fuzzy demand frequency and fuzzy costs [31]. Kefer et al. [32] have analyzed the problem of the classification of potential suppliers in a complex supply chain with respect to three fuzzy criteria. The classification criterion is defined as Euclidean distance between estimated and maximum values of the considered fuzzy criteria. In this paper, the attention of the authors is focused on the RFs classification in PSC.

Contributions of this paper are the following: (1) modeling the severities of consequences and the frequencies of occurrence of RFs using fuzzy sets theory, (2) calculation of overall severity of all consequences for each RF by using FOWA, (3) the fact that determination of risk value occurring due to materialization of each RF is performed by using fuzzy algebra rules, (4) new fuzzy classification model of RFs in PSC (with respect to the practice results, we think that the model developed in this paper describes the real problem significantly better than the literature models), and (5) the fact that determination of the strategy for risk management in PSC may be based on the results of the proposed model, in such manner that the optimal solution should not change over a long period of time.

\section{Proposed Approach}

In this paper, the classification and management of RFs in PSCs are considered, incorporating a new measurement for the classification of the identified RFs in PSCs, based on the distance between fuzzy numbers.

The values of the severities of consequences and the frequency of occurrence of RFs are almost impossible to determine by measurement. These values are obtained on the base of the evidence data and the estimates of the decision maker. It is believed that the decision makers express their estimates much better by linguistic expressions than by precise numbers. In this paper, all existing uncertainties are described by predefined linguistic variables whose values are linguistic terms, that is, words or sentences.

The development of some mathematical areas, such as probability theory, theory of fuzzy sets, and rough theory, enables that uncertainty and imprecision of any kind be sufficiently well described quantitatively. The fuzzy sets theory is widely used in the literature because it (1) resembles human reasoning in its use of approximate information and uncertainty to generate decisions [33] and (2) provides a strict mathematical framework in which vague conceptual phenomena can be precisely and rigorously studied [19]. The number of linguistic variables which describe each treated uncertainty depends on estimation of risk management team. According to literature recommendations, it can be said that the most seven terms can be joined to some linguistic variable. Many authors suggest using triangular functions (TFNs) for describing different uncertainties [34, 35]; because of that, triangular function does not request complex mathematical calculations and by its employment the sufficiently unchangeable results are obtained. There are neither rules nor impediments for determination of domains of fuzzy numbers. In this paper, domains of TFNs are determined into interval [0-1] by analogy to literature results.

\subsection{Definition of Finite Set of Risks Factors and Their Conse-} quences. In this paper, RFs are adopted from Song et al. [4] and they are described in detail in the following.

Demand and supply uncertainty $(i=1)$ is related to situations in which the unreliable and uncertain resources are creating supply chain interruption, thus producing risk that can be explained as uncertainty between supply and demand [36]. The selection of proper suppliers $(i=2)$ could lead to downside of the SC business strategy and its vision for future cooperation as well as lower level of competitiveness, quality, and reputation. Lower responsiveness performance risk factor $(i=3)$ is related to the SC responsiveness performance. Simchi-Levi [37] stated that long chain operative design should be more responsive and with a more reasonable cost than a SC designed as a system with several closed chains of a smaller size. The inflexibility of supply source $(i=4)$ arises from the rate of change and uncertainties in the business environment. The negative implications of this risk occurrence are the increased lead time, dramatic SC performance loss, lower competitiveness, and lower value to customers. The RF marked as a poor quality or process yield at supply source $(i=5)$ is related to supply cost, quality and delivery, suppliers quality certification, supply base reduction, usage of supply contracting, long-term relationships, collaboration with suppliers in product and process development, information exchange, suppliers technical capabilities, and usage of provider rating systems [38]. The operative RF denoted as coordination complexity/effort $(i=6)$ is defined. The most challenging coordination issue is to amplify the concept of coordination from supply chain members to coordination at the level of supply chain as a whole. It enables lower level of coordination complexity in decision-making regarding supply chain logistics activities. Dubey et al. [39] stated that powerful technologies and information $(i=7)$ must 
be considered as an enabler in the strategic framework formulation of sustainable PSC. To decrease internal quality and capabilities, supply chains are associated with lack of sustainable knowledge/technology $(i=8)$.

The volatility of price and cost risk factor $(i=9)$ is tightly correlated with exchange rate and lack of raw materials. Its poor management may cause alleged illegal collection of excess vendor markdown [36]. Inflation and currency exchange rates risk factor $(i=10)$ can include variability and uncertainty in currency exchange rates, economic and political instability, and changes in the regulatory environment. The supply chain is exposed to market share reduction risk ( $i=$ 11 ), that is, to missing out business opportunities that may exist. To avoid market share reduction and to recognize right market signals, supply chain should be responsive to variable market trends and customer requirements. Sodhi et al. [40] stated that supply chain exposure to a reputation loss or brand damage risk is possible $(i=12)$ if the series of undetected faulty products occur in different stages of supply chain and reach the market, which would have to be either reworked or withdrawn. According to Giannakis and Papadopoulos [26], risk management team should consider the RFs that could impose on the natural and social environments, in addition to the operational and economic risk management that is required to make supply chain more sustainable. In automotive supply chain it is understood that supply chain natural disasters risk factor $(i=13)$ may have a higher impact since its occurrence is normally completely unexpected; also it is inherently unknowable in advance and accompanied by grave consequences. To reduce the inefficient use of resources risk factor $(i=14)$ impact, it is necessary to calculate the optimal rate of efficient use of natural resources by an objective analysis and to set short-term targets that will be sufficiently high to begin the process of radically changing the energy system. A large number of different raw materials are used in PSC that may pose a serious threat for environmental pollution causing frequent health problems; therefore it is necessary to keep track of environmental pollution risk factor $(i=15)$ influence and reduce it through reuse and recycling of wastes and making products more resource-efficient. Hazardous waste generation risk $(i=16)$ presents environmental threat that may be caused by inefficient resource use or production of scrap that is potentially very damaging to both the environment and human health. Social group of RFs is defined by Maloni and Brown [41] that are further discussed. The unhealthy/dangerous working environment risk factor $(i=17)$ influences production when working conditions are unhealthy and operations are performed in untrusting workplace, with the use of hazardous materials that threaten employees' health and safety. Violation of human rights ( $i=$ 18 ) is defined with respect to Labor Law. In the automotive PSC industry, the issues that need to be properly addressed are related to environment, fair trade, health and safety, labor and human rights, and community, and they are frequently derived from failure to fulfill social commitment risk $(i=19)$ influence. Violation of business ethics $(i=20)$ is defined by top management.

According to results of good practice and experience of decision makers, it can be said that realization of each
RF may result in the emergence of one or more consequences.

3.2. Choice of Appropriate Linguistic Variables for the Risk Components. The uncertainties into the value of frequency of occurrence of RFs and severities consequences that can appear due to realization of each RF are not measurable variables and depend on subjective judgments of risk management team. In this paper, the existing uncertainties are described by using seven predefined linguistic variables which are modeled by TFNs. These TFNs are presented as follows:

$$
\begin{aligned}
& \text { very low value }(V L)-(x ; 0,0,0.25) \\
& \text { low value }(L)-(x ; 0,0.15,0.3) \\
& \text { medium low value }(M L)-(x ; 0.2,0.35,0.5) \\
& \text { medium value }(M)-(x ; 0.35,0.5,0.65) \\
& \text { medium high value }(M H)-(x ; 0.5,0.65,0.8) \\
& \text { high value }(H)-(x ; 0.7,0.85,1) \\
& \text { very high value }(V H)-(x ; 0.75,1,1)
\end{aligned}
$$

\subsection{Determination of the Relative Importance of Consequences.} The relative importance of each consequence at the level of each identified RF is assumed by risk management team. They base their estimations on the results of good practice and experiences. It is assumed that the relative importance of consequences at the level RF can take some values from interval [0-1] and that they are given as normalized values.

3.4. The Proposed Algorithm. The proposed algorithm can be realized in the following steps which are presented.

Step 1. The first step is calculation of the aggregated and weighted severities of the consequences that arise from the realization of each RF by using FOWA operator [30]:

$$
\widetilde{C}_{i}=\sum_{j=1}^{J_{i}} w_{j i} \cdot \widetilde{v}_{j i}, \quad j=1, \ldots, J_{i} ; i=1, \ldots, I,
$$

where $I$ is the total number of considered RFS, $J_{j}$ is the total number of consequences for $\mathrm{RF} I, i=1, \ldots, I$, which can arise from the realization of each $\operatorname{RF} I, i=1, \ldots, I, w_{j i}$ is the weight of consequence $j, j=1, \ldots, J_{j}$, for RF $I, i=1, \ldots, I$, and $\widetilde{v}_{j i}$ is the TFN describing the value of consequence $j, j=1, \ldots, J_{j}$, under $\operatorname{RF} I, i=1, \ldots, I$.

According to fuzzy algebra rules, severities of all consequences that can appear in PSC (at the level RF $i, i=1, \ldots, I$ ) are TFN, too.

Step 2. The second step is calculation of the risk value that can occur due to materialization $\mathrm{RF} i, \widetilde{R}_{i}, i=1, \ldots, I$ :

$$
\widetilde{R}_{i}=\widetilde{f}_{i} \cdot \widetilde{C}_{i}=\left(r_{i}, \mu_{\widetilde{R}_{i}}\left(r_{i}\right)\right),
$$

where

$$
\mu_{\widetilde{R}_{i}}\left(r_{i}\right)=\min \left(\mu_{\widetilde{f}_{i}}\left(x_{i}\right), \mu_{\widetilde{C}_{i}}\left(x_{i}\right)\right) .
$$


The fuzzy number $\widetilde{R}_{i}$ can be approximated by TFN such as $\widetilde{R}_{i}=\left(l_{i}, m_{i}, u_{i}\right)$. The upper and lower bounds are denoted as $l_{i}, u_{i}$, respectively, and $m_{i}$ is modal value.

Step 3. The third step is determination of the maximal, $\widetilde{R}_{\max }$, and minimal, $\widetilde{R}_{\min }$, values of risk occurring due to the rise from the impact of RF $i, i=1, \ldots, I$.

If the values of frequency of occurrence RF $i, i=1, \ldots I$, have the highest value and all consequences that can arise from the impact of considered $\mathrm{RF} i, i=1, \ldots, I$, have highest value, then risk value arising from the realization of treated $\mathrm{RF}$ is described by an appropriate TFN; $\widetilde{R}_{\max }=(1,1,1)$.

Similarly, the minimal risk value is calculated. It is supposed that (1) the frequency of occurrence RF $i, i=$ $1, \ldots, I$, is very small, almost zero or/and (2) there are no consequences due to materialization $\mathrm{RF} i, i=1, \ldots, I$, or their values are almost zero. Under these assumptions, minimal risk value arising from the materialization of treated $\mathrm{RF}$ is described by TFN; $\widetilde{R}_{\min }=(0,0,0)$.

Step 4. The fourth step is calculation of the distance between TFNs $\widetilde{R}_{i}, i=1, \ldots, I$, and $\widetilde{R}_{\min }$ and $\widetilde{R}_{\max }$, respectively. Denote these distances with $d_{i}^{-}$and $d_{i}^{+}$, respectively. Distance values can be calculated by using different expressions:

(a) Veto concept [20]:

$$
\begin{aligned}
& d_{i}^{-}=\sqrt{\frac{1}{3} \cdot\left[\left(0-l_{i}\right)^{2}+\left(0-m_{i}\right)^{2}+\left(0-u_{i}\right)^{2}\right]}, \\
& d_{i}^{+}=\sqrt{\frac{1}{3} \cdot\left[\left(1-l_{i}\right)^{2}+\left(1-m_{i}\right)^{2}+\left(1-u_{i}\right)^{2}\right]}
\end{aligned}
$$

(b) Procedure developed in Chen and Tenzg [42]:

$$
\begin{aligned}
& d_{i}^{-}=\sqrt{\frac{1}{6} \cdot\left[\left(0-l_{i}\right)^{2}+2 \cdot\left(0-m_{i}\right)^{2}+\left(0-u_{i}\right)^{2}+\left(0-l_{i}\right) \cdot\left(0-m_{i}\right)+\left(0-m_{i}\right) \cdot\left(0-u_{i}\right)\right]}, \\
& d_{i}^{+}=\sqrt{\frac{1}{6} \cdot\left[\left(1-l_{i}\right)^{2}+2 \cdot\left(1-m_{i}\right)^{2}+\left(1-u_{i}\right)^{2}+\left(1-l_{i}\right) \cdot\left(1-m_{i}\right)+\left(1-m_{i}\right) \cdot\left(1-u_{i}\right)\right]}
\end{aligned}
$$

(c) The extension of Grezogorzewski's method based on Hamming distance [43]:

$$
\begin{aligned}
d_{i}^{-} & =\frac{1}{2} \cdot\left[\max \left\{\left(l_{i}-0\right),\left(m_{i}-0\right)\right\}\right. \\
& \left.+\max \left\{\left(m_{i}-0\right),\left(u_{i}-0\right)\right\}\right], \\
d_{i}^{+} & =\frac{1}{2} \cdot\left[\max \left\{\left(1-l_{i}\right),\left(1-m_{i}\right)\right\}\right. \\
& \left.+\max \left\{\left(1-m_{i}\right),\left(1-u_{i}\right)\right\}\right] .
\end{aligned}
$$

The calculated distance values are crisp.

Step 5. The calculated distance values $d_{i}^{+}$are sorted in increasing order. RFs, which are associated with first $a$ sorted values $d_{i}^{+}$, belong to class $\mathrm{A}$. The values of $a$ are determined by results of good practice and experience of risk management team. In this case, the value of $a$ is equal to $30 \%$.

Step 6. Sort the calculated values of $d_{i}^{-}$in increasing order. The value of parameter $c$ is determined by the equivalent way as value of parameter $a$. In this paper, $c=15 \%$.

Step 7. All of the rest of RFs belong to group B.

Step 8 . The eight step is analyzing sensitivity of obtained solutions.

\section{Application of the Proposed Model}

The proposed model is tested on the real-life data from PSC auto industry. RFs in considered PSC are identified according to literature sources [24]. Consequences that can arise from the realization of each identified RF, their relative importance, and severities are obtained by risk management team judgment and presented in Table 1 .

The aggregated value of the consequences severities that arise due to realization of RF ( $i=11)$ is calculated by using FOWA operator [30] (Step 1 of the proposed algorithm):

$$
\begin{aligned}
\widetilde{C}_{11}= & 0.3 \cdot(0.35,0.6,0.65)+0.2 \cdot(0.2,0.35,0.5)+0.1 \\
& \cdot(0,0.15,0.3)+0.4 \cdot(0.5,0.65,0.8), \\
\widetilde{C}_{11}= & (0.345,0.495,0.645) .
\end{aligned}
$$

The risk value that arises due to realization of considered $\mathrm{RF}$ is given (Step 2 of the proposed algorithm):

$$
\begin{aligned}
\widetilde{R}_{11} & =(0.7,0.85,1) \cdot(0.345,0.495,0.645) \\
& =(0.241,0.420,0.645) .
\end{aligned}
$$

Similarly, the risk values that arise due to realization of all other identified RFs are calculated. These obtained risk values are

$$
\begin{aligned}
& \widetilde{R}_{1}=(0.161,0.307,0.498), \\
& \widetilde{R}_{2}=(0.151,0.291,0.476), \\
& \widetilde{R}_{3}=(0.212,0.373,0.580), \\
& \widetilde{R}_{4}=(0,0.069,0.184), \\
& \widetilde{R}_{5}=(0.161,0.305,0.494), \\
& \widetilde{R}_{6}=(0.051,0.141,0.277),
\end{aligned}
$$


TABLE 1: Relative importance and severities of consequences for each risk factor and frequency of occurrence of RF.

\begin{tabular}{|c|c|c|}
\hline RFs & The consequences/the relative importance/severity & Frequency \\
\hline$i=1$ & $\begin{array}{l}\text { Decrease in revenue per order } / 0.3 / M H \text {; layoffs } / 0.35 / M \text {; discontinuation of } \\
\text { investment in development } / 0.25 / M L \text {; termination of cooperation with } \\
\text { subcontractor } / 0.1 / L\end{array}$ & $M H$ \\
\hline$i=2$ & Involvement of existing suppliers $/ 0.45 / \mathrm{M}$; involvement of new suppliers $/ 0.55 / \mathrm{MH}$ & $M$ \\
\hline$i=3$ & $\begin{array}{l}\text { Reduction of the performance of the manufacturing process } / 0.35 / M \text {; reduction of } \\
\text { the performance of the sales process } / 0.25 / \mathrm{MH} \text {; reduction of the performance of the } \\
\text { exploring and development process } / 0.15 / \mathrm{M} \text {; reduction of the performance of the } \\
\text { management process } / 0.25 / \mathrm{MH}\end{array}$ & $\mathrm{MH}$ \\
\hline$i=4$ & $\begin{array}{l}\text { Reduction of the flexibility of the manufacturing process } / 0.3 / M H \text {; reduction of the } \\
\text { flexibility of the sales process } / 0.1 / M L \text {; reduction of the flexibility of the exploring } \\
\text { and development process } / 0.3 / M L \text {; reduction of the flexibility of the management } \\
\text { process } / 0.3 / M L\end{array}$ & $L$ \\
\hline$i=5$ & $\begin{array}{l}\text { Reduction of the quality of the manufacturing process } / 0.5 / H \text {; reduction of the } \\
\text { quality of the sales process } / 0.2 / M \text {; reduction of the quality of the exploring and } \\
\text { development process } / 0.1 / L \text {; reduction of the quality of the management } \\
\text { process } / 0.2 / M L\end{array}$ & $L$ \\
\hline$i=6$ & $\begin{array}{l}\text { Reduction the complexity of the manufacturing process } / 0.4 / M L \text {; reduction of the } \\
\text { complexity of the sales process } / 0.3 / M \text {; reduction of the complexity of the exploring } \\
\text { and development process } / 0.1 / L \text {; reduction of the complexity of the management } \\
\text { process } / 0.2 / M\end{array}$ & $M L$ \\
\hline$i=7$ & Strategic risk/0.2/M; management risk/0.35/M; operational risk/0.45/MH & $M L$ \\
\hline$i=8$ & $\begin{array}{l}\text { Reduction of the technology knowledge } / 0.3 / H \text {; reduction of the marketing } \\
\text { knowledge } / 0.35 / H \text {; reduction of the management and leadership } \\
\text { knowledge } / 0.2 / M L \text {; reduction of maintenance knowledge } / 0.15 / L\end{array}$ & $M$ \\
\hline$i=9$ & $\begin{array}{l}\text { Liquidity reduction } / 0.3 / H \text {; profit decrease } / 0.2 / M H \text {; reduction of } \\
\text { development } / 0.15 / M L \text {; declining of competitiveness } / 0.35 / V H\end{array}$ & $M$ \\
\hline$i=10$ & $\begin{array}{l}\text { Liquidity reduction } / 0.3 / M \text {; profit decrease } / 0.2 / M L \text {; reduction of development } / 0.1 / L \text {; } \\
\text { declining of competitiveness } / 0.4 / M H\end{array}$ & $M L$ \\
\hline$i=11$ & $\begin{array}{l}\text { Liquidity reduction } / 0.3 / M \text {; profit decrease } / 0.2 / M L \text {; reduction of development } / 0.1 / L \text {; } \\
\text { declining of competitiveness } / 0.4 / M H\end{array}$ & $H$ \\
\hline$i=12$ & $\begin{array}{l}\text { Liquidity reduction } / 0.15 / M L \text {; profit decrease } / 0.15 / M \text {; reduction of } \\
\text { development } / 0.15 / M L \text {; declining of competitiveness } / 0.55 / V H\end{array}$ & $M$ \\
\hline$i=13$ & $\begin{array}{l}\text { Consequences of earthquake } / 0.1 / \mathrm{VL} \text {; consequences of fire } / 0.4 / \mathrm{MH} \text {; consequences of } \\
\text { flood } / 0.35 / \mathrm{M} / \text { consequences of hurricane winds/ } 0.15 / \mathrm{VL}\end{array}$ & $V L$ \\
\hline$i=14$ & $\begin{array}{l}\text { Insufficiency of fuels } / 0.4 / M H \text {; insufficiency of metal } / 0.2 / M L \text {; insufficiency of } \\
\text { wood } / 0.2 / M \text {; insufficiency of water } / 0.2 / M H\end{array}$ & $M$ \\
\hline$i=15$ & $\begin{array}{l}\text { Pollution of soil/ } 0.2 / M L \text {; pollution of air/0.3/ML; pollution of food } / 0.2 / M \text {; water } \\
\text { pollution/ } 0.35 / M H\end{array}$ & $M$ \\
\hline$i=16$ & $\begin{array}{l}\text { Pollution of soil/ } 0.5 / V H \text {; pollution of air/0.2/M; pollution of food } / 0.2 / M L \text {; water } \\
\text { pollution } / 0.1 / L\end{array}$ & $M L$ \\
\hline$i=17$ & $\begin{array}{l}\text { Inadequate lighting } / 0.2 / M L \text {; higher level of noise } / 0.3 / M L \text {; higher concentration of } \\
\text { dust } / 0.4 / M \text {; dispersion of working fluid in the working space } / 0.2 / M L\end{array}$ & $M$ \\
\hline$i=18$ & Communication between managers and employees $/ 1 / L$ & $V L$ \\
\hline$i=19$ & Lack of continuous education/1/ML & $M L$ \\
\hline$i=20$ & Unethical behavior of employees $/ 1 / L$ & $L$ \\
\hline
\end{tabular}

$$
\begin{gathered}
\widetilde{R}_{7}=(0.077,0.188,0.343), \\
\widetilde{R}_{8}=(0.152,0.292,0.477), \\
\widetilde{R}_{9}=(0.200,0.390,0.575), \\
\widetilde{R}_{10}=(0.111,0.283,0.472),
\end{gathered}
$$$$
\widetilde{R}_{12}=(0.184,0.365,0.518) \text {, }
$$$$
\widetilde{R}_{13}=(0,0,0.152) \text {, }
$$$$
\widetilde{R}_{14}=(0.143,0.280,0.461) \text {, }
$$$$
\widetilde{R}_{15}=(0.112,0.235,0.403) \text {, }
$$ 


$$
\begin{aligned}
& \widetilde{R}_{16}=(0.097,0.239,0.380), \\
& \widetilde{R}_{17}=(0.091,0.205,0.364), \\
& \widetilde{R}_{18}=(0,0,0.075), \\
& \widetilde{R}_{19}=(0.040,0.122,0.250), \\
& \widetilde{R}_{20}=(0,0.075,0.195) .
\end{aligned}
$$

Thereafter, distances among TFNs are calculated by which the risk values that arise from the realization of RF $(i=11)$ are described as $\widetilde{R}_{\min }$ and $\widetilde{R}_{\max }$ (Steps 3-4 of the proposed algorithm): (a)

$$
\begin{aligned}
& d_{11}^{-} \\
& =\sqrt{\frac{1}{3} \cdot\left[(0-0.241)^{2}+(0-0.420)^{2}+(0-0.645)^{2}\right]} \\
& =0.4657, \\
& d_{11}^{+} \\
& =\sqrt{\frac{1}{3} \cdot\left[(1-0.241)^{2}+(1-0.420)^{2}+(1-0.645)^{2}\right]} \\
& =0.5884
\end{aligned}
$$

$$
\begin{aligned}
d_{11}^{-} & =\sqrt{\frac{1}{6} \cdot\left[(0-0.241)^{2}+2 \cdot(0-0.420)^{2}+(0-0.645)^{2}+(0-0.241) \cdot(0-0.420)+(0-0.420) \cdot(0-0.645)\right]} \\
& =0.4470 \\
d_{11}^{-} & =\sqrt{\frac{1}{6} \cdot\left[(1-0.241)^{2}+2 \cdot(1-0.420)^{2}+(1-0.645)^{2}+(1-0.241) \cdot(1-0.420)+(1-0.420) \cdot(1-0.645)\right]} \\
& =0.5804
\end{aligned}
$$

(c)

$$
\begin{aligned}
d_{11}^{-} & =\frac{1}{2} \cdot[\max \{(0.241-0),(0.42-0)\} \\
& +\max \{(0.42-0),(0.645-0)\}]=0.5325, \\
d_{11}^{+} & =\frac{1}{2} \cdot[\max \{(1-0.241),(1-0.42)\} \\
& +\max \{(1-0.42),(1-0.645)\}]=0.5895 .
\end{aligned}
$$

The ranks of identified RFs, which were obtained by the proposed algorithm (Steps 5-7), are presented in Tables 2-7.

According to obtained results in Tables 2-6, RFs that have the highest influence onto business of PSC are described. These results are illustrated in Figure 1 (Step 8 of the proposed algorithm).

Based on the obtained results, shown in Figure 1, it can be concluded that solution is not changed in dependence on used measure distance. It indicates stability of solutions obtained by applying the developed model. Risk management team has to define improved business plan of PSC with respect to RFs. The results of measure taken in order to lead to decreases of influence of RFs are monitored and evaluated in anticipated time intervals. According to the obtained results, risk management team should take corrective measure. In the following, RFs that belong to group A and actions that should be taken in order to reduce influences of these RFs are shown.

According to the results obtained (see Figure 1), it can be concluded that the highest priority is given to RFs of demand and supply uncertainty $(i=1)$ and lack of sustainable knowledge/technology $(i=8)$. Reducing uncertainty demand and supply $(i=1)$ can be achieved if one makes a detailed marketing analysis by using direct marketing method and by applying the business to business concept. To minimize the impact of RF $(i=8)$, it is necessary to determine the priority of lacking knowledge of employees. The next step for priority skills is determination of the strategy and tactics for employees to acquire these skills. This piece of knowledge can be acquired through either bringing new staff or training staff. Applying management initiatives such as improving relations with customers, the development of new brands, and improving the quality of the product can reduce the impact of brand reputation loss or damage $(i=12)$. Improving the responsiveness lower performance $(i=3)$ may be achieved through (a) improving the performance of the business processes and products by using the new and the standard methods and (b) the improvement of knowledge and motivation for their employees through the new corporate culture. Reducing the volatility of price and cost $(i=9)$ can be achieved through greater cost control inputs by applying cost management system and through greater cost control process by applying Activity Based Costing. Application of marketing measure, which leads to creation of new and improvement of innovation, can lead to decreases in market share reduction $(i=11)$.

The results that are shown in Tables 3-7 are illustrated in Figure 2. 
TABLE 2: Rank of identified RFs with respect to distance values [20] $d_{i}^{+}$.

\begin{tabular}{lc}
\hline Risk factors & $d_{i}^{+}$ \\
\hline$i=11$ & 0.5884 \\
$i=3$ & 0.6299 \\
$i=9$ & 0.6305 \\
$i=12$ & 0.6586 \\
$i=1$ & 0.6919 \\
$i=8$ & 0.6919 \\
$i=5$ & 0.6936 \\
$i=2$ & 0.7066 \\
$i=14$ & 0.7173 \\
$i=10$ & 0.7265 \\
$i=15$ & 0.7594 \\
$i=16$ & 0.7700 \\
$i=17$ & 0.7880 \\
$i=7$ & 0.8048 \\
$i=6$ & 0.8488 \\
$i=19$ & 0.8670 \\
$i=20$ & 0.9135 \\
$i=4$ & 0.9188 \\
$i=13$ & 0.9520 \\
$i=18$ & 0.9756
\end{tabular}

TABLE 3: Rank of identified RFs with respect to distance values [20] $d_{i}^{-}$.

\begin{tabular}{lc}
\hline Risk factors & $d_{i}^{-}$ \\
\hline$i=18$ & 0.0433 \\
$i=13$ & 0.0878 \\
$i=4$ & 0.1135 \\
$i=20$ & 0.1206 \\
$i=19$ & 0.1623 \\
$i=6$ & 0.1819 \\
$i=7$ & 0.2302 \\
$i=17$ & 0.2468 \\
$i=16$ & 0.2657 \\
$i=15$ & 0.2770 \\
$i=14$ & 0.3222 \\
$i=10$ & 0.3241 \\
$i=2$ & 0.3337 \\
$i=5$ & 0.3478 \\
$i=1$ & 0.3503 \\
$i=8$ & 0.3503 \\
$i=12$ & 0.3809 \\
$i=3$ & 0.4165 \\
$i=11$ & 0.4174 \\
& $=0.4657$
\end{tabular}

With respect to obtained results, it can be concluded that the stability of solutions is achieved if one observes RFs from group C. These RFs are inflexibility of supply source $(i=4)$, natural disasters $(i=13)$, and violation of human
TABLE 4: Rank of identified RFs with respect to distance values [42] $d_{i}^{+}$.

\begin{tabular}{lc}
\hline Risk factors & $d_{i}^{+}$ \\
\hline$i=9$ & 0.6208 \\
$i=3$ & 0.6246 \\
$i=1$ & 0.6279 \\
$i=8$ & 0.6279 \\
$i=11$ & 0.6378 \\
$i=12$ & 0.6492 \\
$i=5$ & 0.6905 \\
$i=2$ & 0.7041 \\
$i=14$ & 0.7149 \\
$i=10$ & 0.7203 \\
$i=15$ & 0.7584 \\
$i=4$ & 0.7617 \\
$i=16$ & 0.7656 \\
$i=17$ & 0.7877 \\
$i=7$ & 0.8047 \\
$i=6$ & 0.8500 \\
$i=19$ & 0.8686 \\
$i=13$ & 0.9013 \\
$i=20$ & 0.9155 \\
$i=18$ & 0.9815 \\
\hline &
\end{tabular}

TABLE 5: Rank of identified RFs with respect to distance values [42] $d_{i}^{-}$.

\begin{tabular}{ll}
\hline Risk factors & $d_{i}^{-}$ \\
\hline$i=18$ & 0.0306 \\
$i=13$ & 0.0621 \\
$i=4$ & 0.0850 \\
$i=20$ & 0.1032 \\
$i=19$ & 0.1468 \\
$i=6$ & 0.1660 \\
$i=7$ & 0.2134 \\
$i=17$ & 0.2303 \\
$i=15$ & 0.2419 \\
$i=16$ & 0.2523 \\
$i=14$ & 0.2830 \\
$i=10$ & 0.3056 \\
$i=1$ & 0.3084 \\
$i=8$ & 0.3084 \\
$i=2$ & 0.3165 \\
$i=5$ & 0.3306 \\
$i=12$ & 0.3708 \\
$i=3$ & 0.3989 \\
$i=9$ & 0.4035 \\
$i=11$ & 0.4470 \\
\hline
\end{tabular}

rights $(i=18)$, and they have the least impact on the effectiveness of operations of PSC. Managing these RFs is based on the strict application of procedures prescribed by state or local self-government and does not affect the increase 
TABLE 6: Rank of identified RFs with respect to distance values [43] $d_{i}^{+}$.

\begin{tabular}{ll}
\hline Risk factors & $d_{i}^{+}$ \\
\hline$i=11$ & 0.6695 \\
$i=9$ & 0.7050 \\
$i=3$ & 0.7075 \\
$i=12$ & 0.7255 \\
$i=1$ & 0.7660 \\
$i=8$ & 0.7660 \\
$i=5$ & 0.7670 \\
$i=2$ & 0.7790 \\
$i=14$ & 0.7885 \\
$i=10$ & 0.8030 \\
$i=15$ & 0.8265 \\
$i=16$ & 0.8320 \\
$i=17$ & 0.8520 \\
$i=7$ & 0.8675 \\
$i=6$ & 0.9040 \\
$i=19$ & 0.9190 \\
$i=20$ & 0.9625 \\
$i=4$ & 0.9655 \\
$i=13$ & 1
\end{tabular}

TABLE 7: The rank of identified RFs with respect to distance values [43] $d_{i}^{-}$.

\begin{tabular}{ll}
\hline Risk factors & $d_{i}^{-}$ \\
\hline$i=18$ & 0.0375 \\
$i=13$ & 0.0760 \\
$i=4$ & 0.1265 \\
$i=20$ & 0.1350 \\
$i=19$ & 0.1860 \\
$i=6$ & 0.2090 \\
$i=7$ & 0.2655 \\
$i=17$ & 0.2845 \\
$i=16$ & 0.3095 \\
$i=15$ & 0.3190 \\
$i=14$ & 0.3705 \\
$i=10$ & 0.3775 \\
$i=2$ & 0.3835 \\
$i=5$ & 0.3995 \\
$i=1$ & 0.4025 \\
$i=8$ & 0.4025 \\
$i=12$ & 0.4415 \\
$i=3$ & 0.4765 \\
$i=11$ & 0.4825 \\
\hline & $=9.5325$ \\
\hline & $=1$
\end{tabular}

in management costs. For example, the management of the PSC should respect the methods of reaction in the case of natural disasters, which are defined in the plan of prevention.

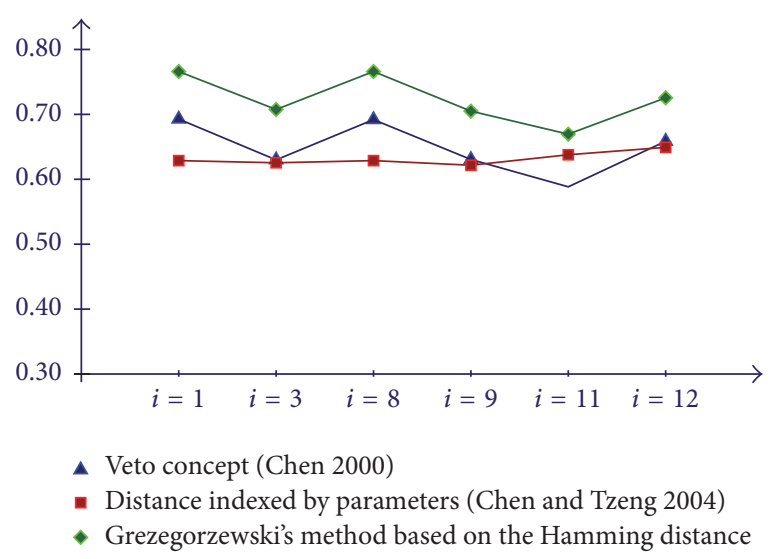

FIGURE 1: Change of RFs of group A in dependence on used measure distance.

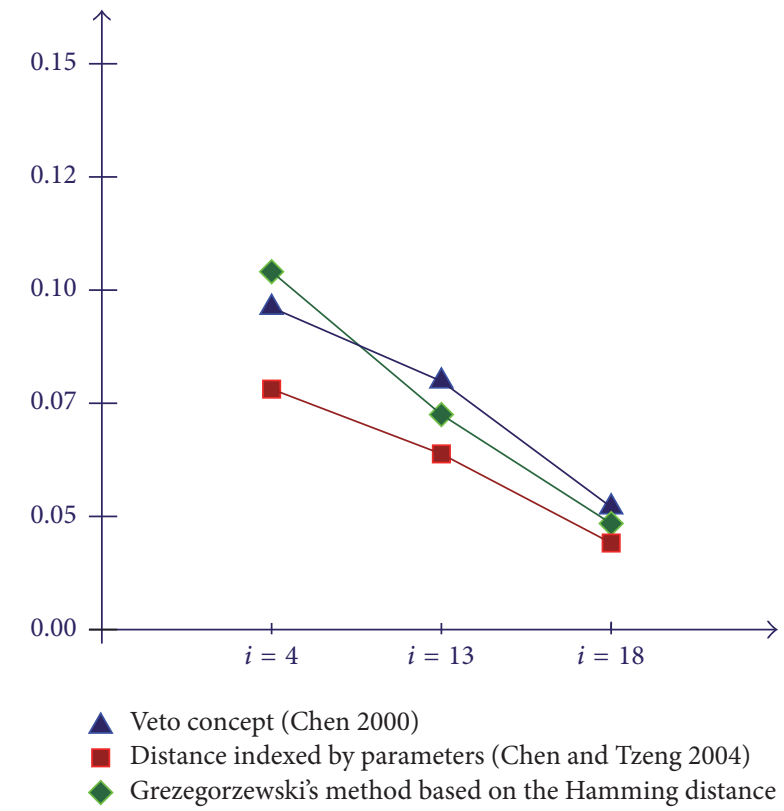

FIgURE 2: Change of RFs of group C in dependence on used measure distance.

Also, the demands of law to employees must be strictly applied in the PSC.

The rest of considered RFs belong to group B, which have a mean significance for risk management team. Measures which should be taken to reduce the impact of these RFs are not that expensive. These measures are increasing the legal security of vendor in the contracting process which leads to reduction in failure to select the right suppliers $(i=2)$, improvement of communication channels which significantly impacts coordination complexity/effort $(i=6)$, typing of ICT equipment which leads to reduction of IT and information sharing risks $(i=7)$, improvement of the process of resource planning in a PSC which can reduce inefficient use of resources $(i=14)$, using corresponding filters, environmental pollution $(i=15)$, and hazardous waste generation $(i=16)$ which can be reduced, although staff are educated and motivated to 
handle the waste, particularly hazardous waste, application of defined procedures for the safe work at a workplace which leads to significant reduction of unhealthy/dangerous working environment $(i=17)$, application of the rules of procedures in accordance with the new business ethics which leads to reduction of violation of business ethics $(i=20)$.

\section{Conclusion}

Management practice of PSC shows that assessment and reduction of influence of RFs lead to significant improvement and safety of business processes, and represents one of the most relevant issues of competitiveness and sustainability. The identification of RFs is based on literature source [4]. In order to reduce the cost and decision time and simultaneously increase effectiveness of risk supply management, one should classify the identified RFs according to their priorities. It is assumed that application of analytic methods in determining the priority of RFs is better than applying intuitive decisionmaking methods. It may be suggested that each solution obtained in an exact way is less encumbered by the subjective views of decision makers, so this could make it more accurate.

The main contribution of the paper may be seen in the domain of the mathematical model development and its practical implications.

In the domain of the mathematical model development, the most significant contributions are as follows: (a) all uncertainties in the severity of consequences and frequency of occurrence of RFs are described by linguistic variable. It is considered that decision makers much better expressed their evaluation by using linguistic statement than by using precious number. These linguistic expressions are modeled by TFNs that offer a good compromise between descriptive power and computational simplicity; (b) materialization of identified RFs leads to generation of more consequences that have different relative importance which are assessed by risk management team. Decision makers base their evaluations on their experience and to degree of implementation ISO 31000; (c) the aggregated overall severity of all consequences for each RF is calculated by using FOWA operator [30]; (d) calculation of value of risks generated by materialization of each RF is based on applying fuzzy algebra rules [19]; and (e) classification of identified RFs is given by using the proposed fuzzy model.

The proposed model is flexible: (1) all changes, as those in consequence severities or frequencies values and the membership function shape of fuzzy numbers, can be easily and quickly incorporated into the model and (2) the proposed model could be modified for solving risk problems in different types of organizations, depending on the their size and branch of economy.

In the domain of practical implications, the significant contribution is the development of safety of critical systems.

Besides the advantages, the proposed model has certain constraints. The authors think that the main constraint of the proposed model is determination of consequences and evaluation of their severities. The priorities of RFs are changed when the number or relative importance of consequences is changed.
The focus of future research should be set on a case study of a large sample of PSCs in the automotive industry in order to fully validate the proposed model. Also, the development of software, based on the proposed model with additional functionalities for better risk management, should be performed.

\section{Conflicts of Interest}

The authors declare that there are no conflicts of interest regarding the publication of this paper.

\section{References}

[1] J. G. March and Z. Shapira, "Managerial perspectives on risk and risk taking," Management Science, vol. 33, no. 1, pp. 1404-1418, 1987.

[2] D. D. Wu and D. L. Olson, "Computational simulation and risk analysis: an introduction of state of the art research," Mathematical and Computer Modelling, vol. 58, no. 9-10, pp. 1581-1587, 2013.

[3] P. Antunes and H. Mourão, "Resilient business process management: Framework and Services," Expert Systems with Applications, vol. 38, no. 2, pp. 1241-1254, 2011.

[4] W. Song, X. Ming, and H. Liu, "Identifying critical risk factors of sustainable supply chain management: A rough strengthrelation analysis method," Journal of Cleaner Production, vol. 143, pp. 100-115, 2017.

[5] A. C. Caputo, P. M. Pelagagge, and P. Salini, "A multicriteria knapsack approach to economic optimization of industrial safety measures," Safety Science, vol. 51, no. 1, pp. 354-360, 2013.

[6] C. Mabrouki, F. Bentaleb, and A. Mousrij, "A decision support methodology for risk management within a port terminal," Safety Science, vol. 63, pp. 124-132, 2014.

[7] A. Bounit, E. Irhirane, N. Bourquia, and R. Benmoussa, "Design of a fuzzy model that integrates hygiene, safety, and environment systems for the assessment of the overall risk of machines," Proceedings of the Institution of Mechanical Engineers, Part O: Journal of Risk and Reliability, vol. 230, no. 4, pp. 378-390, 2016.

[8] M. M. van der Voort, A. J. J. Klein, M. de Maaijer, A. C. van den Berg, J. R. van Deursen, and N. H. A. Versloot, "A quantitative risk assessment tool for the external safety of industrial plants with a dust explosion hazard," Journal of Loss Prevention in the Process Industries, vol. 20, no. 4-6, pp. 375-386, 2007.

[9] P. Stanojevic, B. Orlic, M. Misita, N. Tatalovic, and G. B. Lenkey, "Online monitoring and assessment of emerging risk in conventional industrial plants: Possible way to implement integrated risk management approach and KPIs," Journal of Risk Research, vol. 16, no. 3-4, pp. 501-512, 2013.

[10] C. Eckert and N. Gatzert, "Modeling operational risk incorporating reputation risk: an integrated analysis for financial firms," Insurance: Mathematics and Economics, vol. 72, pp. 122-137, 2017.

[11] N. Gatzert and A. Kolb, "Risk measurement and management of operational risk in insurance companies from an enterprise perspective," Journal of Risk and Insurance, vol. 81, no. 3, pp. 683-708, 2014.

[12] M. J. Djapan, D. P. Tadic, I. D. Macuzic, and P. D. Dragojovic, "A new fuzzy model for determining risk level on the workplaces in manufacturing small and medium enterprises," Proceedings of the Institution of Mechanical Engineers, Part O: Journal of Risk and Reliability, vol. 229, no. 5, pp. 456-468, 2015. 
[13] A. Pinto, "QRAM a qualitative occupational safety risk assessment model for the construction industry that incorporate uncertainties by the use of fuzzy sets," Safety Science, vol. 63, pp. 57-76, 2014.

[14] M. Fera and R. Macchiaroli, "Appraisal of a new risk assessment model for SME," Safety Science, vol. 48, no. 10, pp. 1361-1368, 2010.

[15] H. Akyildiz and A. Mentes, "An integrated risk assessment based on uncertainty analysis for cargo vessel safety," Safety Science, vol. 92, pp. 34-43, 2017.

[16] S. A. Torabi, R. Giahi, and N. Sahebjamnia, "An enhanced risk assessment framework for business continuity management systems," Safety Science, vol. 89, pp. 201-218, 2016.

[17] S. Basu, Plant Hazard Analysis and Safety Instrumentation Systems, Academic Press is an imprint of Elsevier, Oxford, UK, 2017.

[18] B. Gallagher, P. Case, R. Creel, S. Kushner, and R. Williams, A taxonomy of operational risks, Carnegie Mellon University, Pittsburgh, Pa, USA, 2005.

[19] H. Zimmermann, Fuzzy set theory-and its applications, Springer Science and Business Media, Amsterdam, Nederland, 1st edition, 2001.

[20] C. Chen, "Extensions of the TOPSIS for group decision-making under fuzzy environment," Fuzzy Sets and Systems, vol. 114, no. 1, pp. 1-9, 2000.

[21] R. M. Stulz, "Rethinking Risk Management," Journal of Applied Corporate Finance, vol. 9, no. 3, pp. 8-25, 1996.

[22] "Risk management-Principles and guidelines," 31000:2009. ISO 31000:2009, International Standards Organisation, Geneva, Switzerland, 2017.

[23] R. J. Chapman, Simple tools and techniques for enterprise risk management, John Wiley \& Sons, Ltd., Chichester, UK, 1st edition, 2012.

[24] S. Chopra and S. Sodhi, "Managing risk to avoid supply-chain breakdown," MIT Sloan management review, vol. 46, no. 1, 53 pages, 2004.

[25] E. Hofmann, "Natural hedging as a risk prophylaxis and supplier financing instrument in automotive supply chains," Supply Chain Management, vol. 16, no. 2, pp. 128-141, 2011.

[26] M. Giannakis and T. Papadopoulos, "Supply chain sustainability: a risk management approach," International Journal of Production Economics, vol. 171, pp. 455-470, 2016.

[27] T. Kontogiannis, V. Leopoulos, and N. Marmaras, "A comparison of accident analysis techniques for safety-critical man-machine systems," International Journal of Industrial Ergonomics, vol. 25, no. 4, pp. 327-347, 2000.

[28] J. M. Woodruff, "Consequence and likelihood in risk estimation: a matter of balance in UK health and safety risk assessment practice," Safety Science, vol. 43, no. 5-6, pp. 345-353, 2005.

[29] C. Hess, "The impact of the financial crisis on operational risk in the financial services industry: empirical evidence," The Journal of Operational Risk, vol. 6, no. 1, pp. 23-35, 2011.

[30] J. M. Merig, M. Casanovas, and J. M. Merigó, "Using fuzzy numbers in heavy aggregation operators," International Journal of Information Technology, vol. 4, no. 3, pp. 177-182, 2008.

[31] J. Puente, D. de la Fuente, P. Priore, and R. Pino, "Abc classification with uncertain data. A fuzzy model vs. a probabilistic model," Applied Artificial Intelligence, vol. 16, no. 6, pp. 443-456, 2002.

[32] P. Kefer, D. D. Milanovic, M. Misita, and A. Zunjic, "Fuzzy multicriteria $\mathrm{ABC}$ supplier classification in global supply chain,"
Mathematical Problems in Engineering, Article ID 9139483, Art. ID 9139483, 11 pages, 2016.

[33] C. Kahraman, T. Ertay, and G. Büyüközkan, "A fuzzy optimization model for QFD planning process using analytic network approach," European Journal of Operational Research, vol. 171, no. 2, pp. 390-411, 2006.

[34] F. Torfi, R. Z. Farahani, and S. Rezapour, "Fuzzy AHP to determine the relative weights of evaluation criteria and Fuzzy TOPSIS to rank the alternatives," Applied Soft Computing Journal, vol. 10, no. 2, pp. 520-528, 2010.

[35] A. Aleksić, M. Stefanović, D. Tadić, and S. Arsovski, "A fuzzy model for assessment of organization vulnerability," Measurement, vol. 51, no. 1, pp. 214-223, 2014.

[36] O. Tang and S. Nurmaya Musa, "Identifying risk issues and research advancements in supply chain risk management," International Journal of Production Economics, vol. 133, no. 1, pp. 25-34, 2011.

[37] D. Simchi-Levi, Operations rules, MIT Press, Cambridge, Mass, USA, 1st edition, 2010.

[38] R. Tummala and T. Schoenherr, "Assessing and managing risks using the Supply Chain Risk Management Process (SCRMP)," Supply Chain Management, vol. 16, no. 6, pp. 474-483, 2011.

[39] R. Dubey, A. Gunasekaran, T. Papadopoulos, S. J. Childe, K. Shibin, and S. F. Wamba, "Sustainable supply chain management: framework and further research directions," Journal of Cleaner Production, vol. 142, pp. 1119-1130, 2017.

[40] M. S. Sodhi, B.-G. Son, and C. S. Tang, "Researchers' perspectives on supply chain risk management," Production and Operations Management, vol. 21, no. 1, pp. 1-13, 2012.

[41] M. J. Maloni and M. E. Brown, "Corporate social responsibility in the supply chain: An application in the food industry," Journal of Business Ethics, vol. 68, no. 1, pp. 35-52, 2006.

[42] M. F. Chen and G. H. Tzeng, "Combining grey relation and TOPSIS concepts for selecting an expatriate host country," Mathematical and Computer Modelling, vol. 40, no. 13, pp. 14731490, 2004.

[43] J. H. Park, K. M. Lim, J. S. Park, and Y. C. Kwun, "Distances between interval-valued intuitionistic fuzzy sets," Journal of Physics: Conference Series, vol. 96, no. 1, Article ID 012089, 2008. 


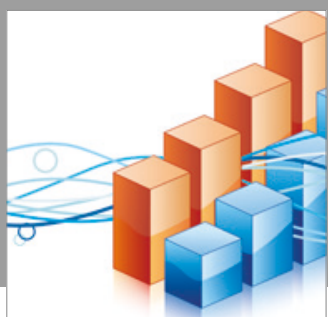

Advances in

Operations Research

vatersals

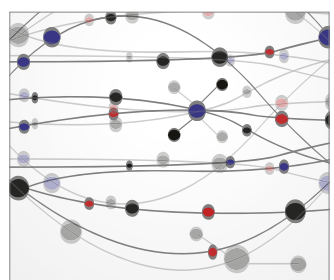

\section{The Scientific} World Journal
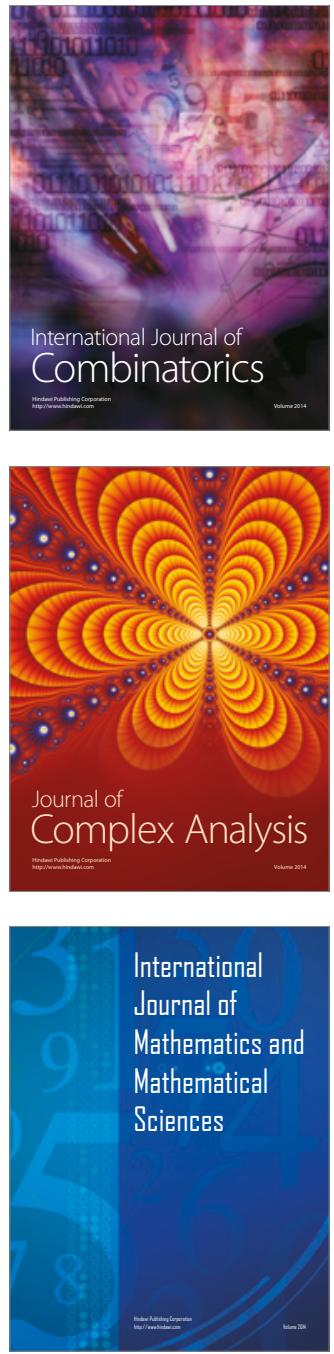
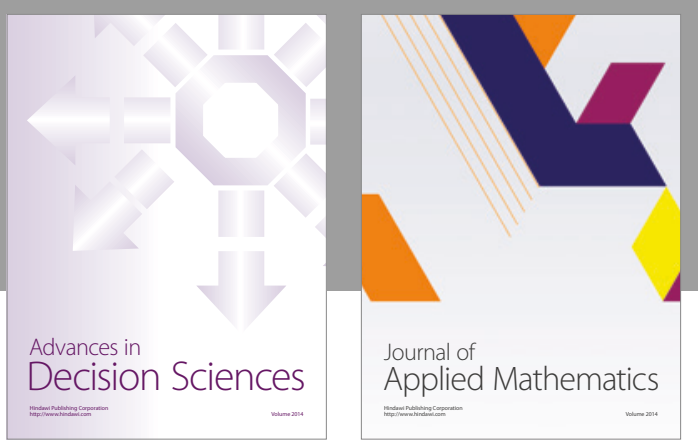

Algebra

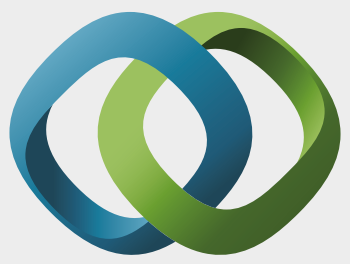

\section{Hindawi}

Submit your manuscripts at

https://www.hindawi.com
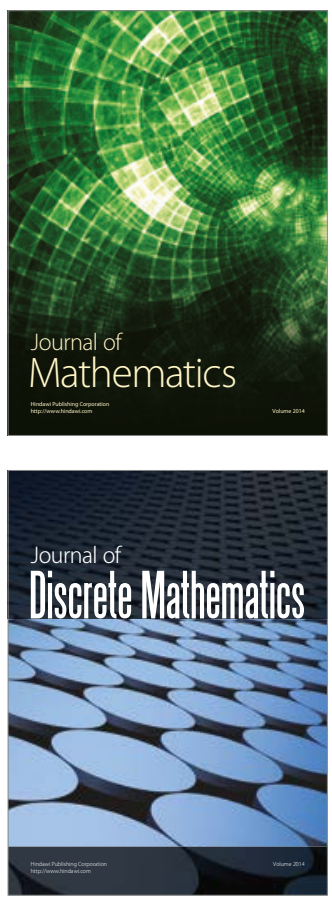

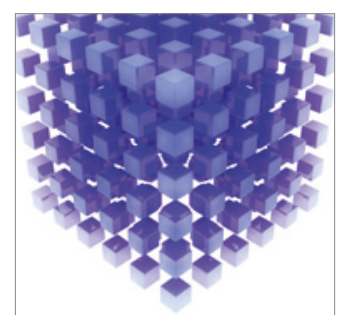

Mathematical Problems in Engineering
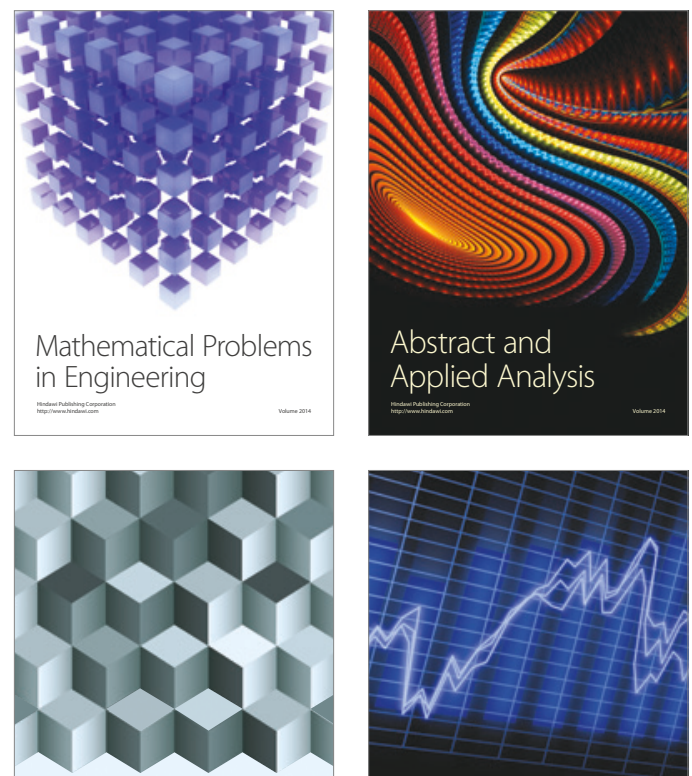

Journal of

Function Spaces

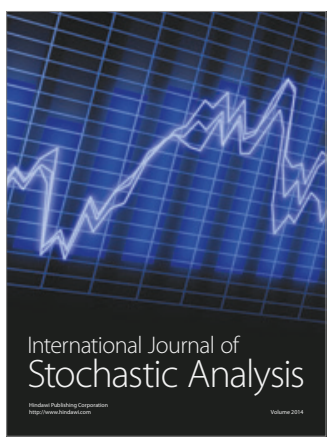

Probability and Statistics
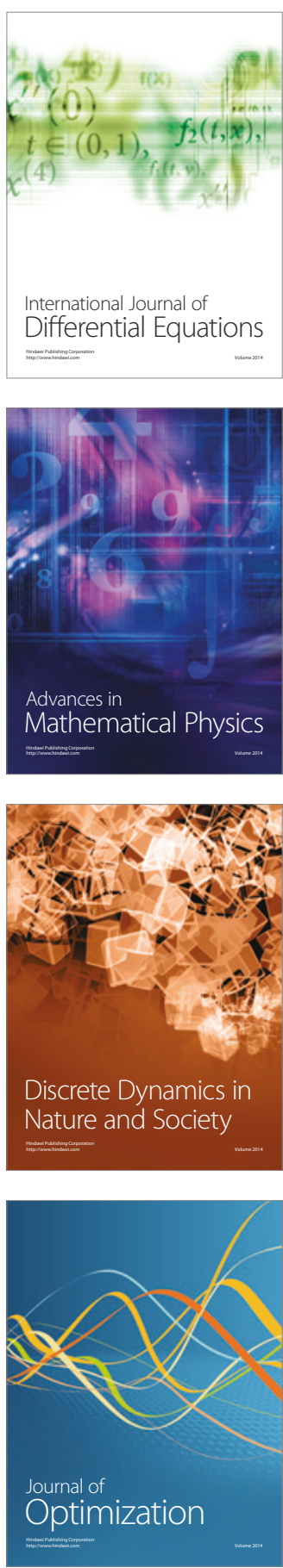